\title{
CORRESPONDENCE
}

\section{Intraoperative Computed Tomography Ensures Inferior Alveolar Nerve Safety During Orthognathic Surgery}

Damage to the inferior alveolar nerve (IAN) is a major complication of orthognathic surgical procedures and accounts for the majority of postoperative complications of bilateral sagittal split osteotomy (BSSO). ${ }^{1}$ Inferior alveolar nerve injury during surgery largely results from manipulation of the nerve or structures surrounding the nerve, or from direct injury to the nerve during the operation, ${ }^{2}$ and because of piercing of the nerve with osteosynthesis screws (Fig. 1A).

Here we elucidate the importance of intraoperative computed tomography $(\mathrm{CT})$ as an aid for ensuring IAN safety during BSSO by confirming that there is no piercing of the IAN by osteosynthesis screws during the procedure.

This study included 2 patients who underwent BSSO. Both patients were treated for orthognathic reasons. The BSSO surgical technique was previously described in detail. ${ }^{3}$

The patients underwent $\mathrm{CT}$ with an Artis Zeego multiaxis system (Siemens AG, Healthcare Sector, Henkestrasse 127 D-91052 Erlangen, Germany) at the beginning of the operation and immediately after placement of the osteosynthesis plates and screws (Fig. 1B).

Immediate image generation enabled visualization of the state of the mandibular bone and the depth of the screws in the region of the osteosynthesis (Fig. 1C).

Proper localization of the IAN before the procedure is an essential preventive step. Although conventional radiography is most commonly used for this localization, cone-beam computed tomography (CBCT) enables nerve assessment in 3 dimensions. New surgical techniques such as safe surgical access to the mandibular nerve at the infratemporal fossa, ${ }^{4}$ as well as modifications of existing surgical techniques, for example to control the lower border cut, are important for reducing the occurrence of postoperative complications of BSSO.

Because severe nerve injuries are often the result of drilling too deep (past the bone and into the nerve) or placing the osteosynthesis screw itself upon a nerve (Fig. 1A), the accurate measurement of available bone can assist the surgeon in choosing the correct osteosynthesis screw length, thereby preventing over-penetration. The use of intraoperative CT during BSSO allows intraoperative evaluation of osteosynthesis screw penetration and depth, making it possible to modify the treatment if necessary.

Based on the aforementioned advantages, the use of intraoperative $\mathrm{CT} / \mathrm{CBCT}$ is beneficial and thus recommended. More common use could be expected with technological improvements leading to an affordable, less bulky, and easier to handle intraoperative $\mathrm{CT} / \mathrm{CBCT}$ equipment.

AQ1

AQ2
Jimoh Olubanwo Agbaje, BDS, DMD OMFS-IMPATH Research Group Department of Imaging and Pathology Faculty of Medicine Catholic University Leuven, Belgium

Oral and Maxillofacial Surgery St. John's Hospital Genk, Belgium
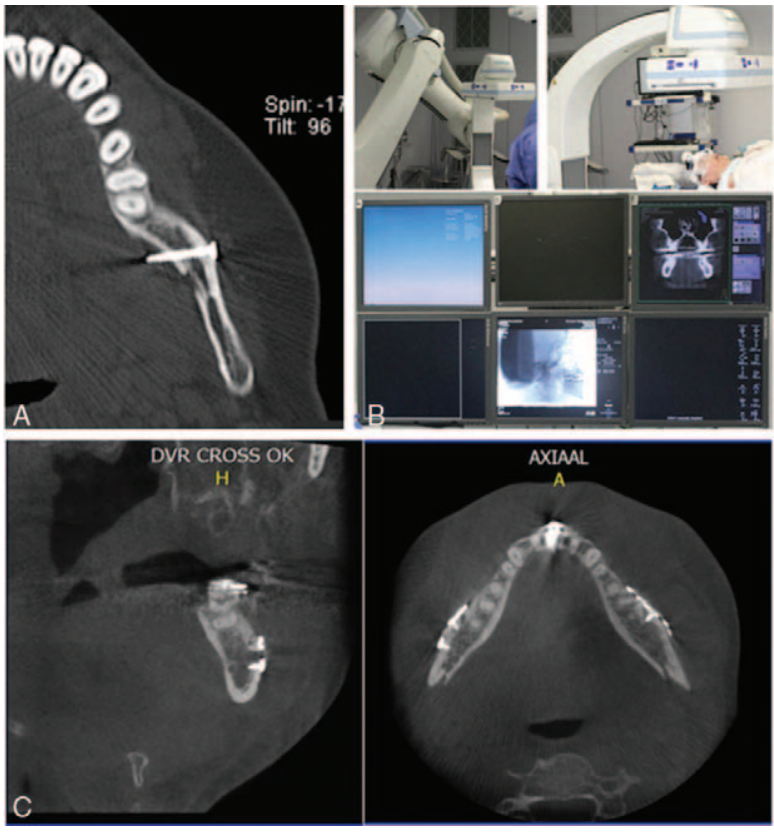

FIGURE 1. A, Inferior alveolar nerve damage by osteosynthesis screw during BSSO. B, Artis Zeego CT machine. C, Immediate CT image generated in the region of the osteosynthesis.

Ahmed S. Salem, BDS, MSc OMFS-IMPATH Research Group Department of Imaging and Pathology Faculty of Medicine Catholic University Leuven, Belgium

Oral and Maxillofacial Surgery Department Faculty of Dentistry Mansoura University, Egypt

Ivo Lambrichts, DDS, $\mathrm{PhD}$ Faculty of Medicine Hasselt University Diepenbeek, Belgium

Reinhilde Jacobs, DDS, PhD OMFS-IMPATH Research Group Department of Imaging and Pathology Faculty of Medicine Catholic University Leuven, Belgium

Constantinus Politis, MD, $\mathrm{PhD}$ OMFS-IMPATH Research Group Department of Imaging and Pathology Faculty of Medicine Catholic University Leuven, Belgium

Faculty of Medicine Hasselt University Diepenbeek, Belgium stan@politis.be 


\section{REFERENCES}

1. D'Agostino A, Trevisiol L, Gugole F, et al. Complications of orthognathic surgery: the inferior alveolar nerve. J Craniofac Surg 2010;21:1189-1195

2. Yoshioka I, Tanaka T, Khanal A, et al. Relationship between inferior alveolar nerve canal position at mandibular second molar in patients with prognathism and possible occurrence of neurosensory disturbance after sagittal split ramus osteotomy. J Oral Maxillofac Surg 2010;68:30223027

3. Falter B, Schepers S, Vrielinck L, et al. Occurrence of bad splits during sagittal split osteotomy. Oral Surg Oral Med Oral Pathol Oral Radiol Endod 2010;110:430-435

4. Agbaje JO, Sun Y, Lambrichts I, et al. Safe surgical access to the mandibular nerve at the infratemporal fossae. J Craniofac Surg 2014;25: 1454-1457 


\section{SCS}

Manuscript No. 0301

\section{Journal of Craniofacial Surgery}

Typeset by Thomson Digital for Lippincott Williams \& Wilkins

Dear Author,

During the preparation of your manuscript for typesetting, some queries have arisen. These are listed below. Please check your typeset proof carefully and mark any corrections in the margin as neatly as possible or compile them as a separate list. This form should then be returned with your marked proof/list of corrections to the Production Editor.

\section{QUERIES: to be answered by AUTHOR}

\begin{tabular}{|l|l|l|}
\hline QUERY NO. & \multicolumn{1}{c|}{ QUERY DETAILS } & RESPONSE \\
\hline$<$ AQ1 $>$ & $\begin{array}{l}\text { Please check the affiliations for } \\
\text { correctness. }\end{array}$ \\
$<$ AQ2 $>\quad$ & $\begin{array}{l}\text { As per style, only 2 degrees are allowed. } \\
\text { Please check. }\end{array}$
\end{tabular}

